\title{
Brain is an endocrine organ through secretion and nuclear transfer of parathymosin
}

Bin $\mathrm{Yu}^{1,2,3, \star}$, Yizhe Tang ${ }^{1,2,3, *}$, Dongsheng Cai ${ }^{1,2,3}$ (1)

This study reports that parathymosin (PTMS) is secreted by hypothalamic stem/progenitor cells (htNSC) to inhibit senescence of recipient cells such as fibroblasts. Upon release, PTMS is rapidly transferred into the nuclei of various cell types, including neuronal GT1-7 cells and different peripheral cells, and it is effectively transferred into neuronal nuclei in various brain regions in vivo. Notably, brain neurons also produce and release PTMS, and because neuronal populations are large, they are important for maintaining PTMS in the cerebrospinal fluid which is further transferable into the blood. Compared with several other brain regions, the hypothalamus is stronger for long-distance PTMS transfer, supporting a key hypothalamic role in this function. In physiology, aging is associated with declines in PTMS production and transfer in the brain, and ptms knockdown in the hypothalamus versus hippocampus were studied showing different contributions to neurobehavioral physiology. In conclusion, the brain is an endocrine organ through secretion and nuclear transfer of PTMS, and the hypothalamus-brain orchestration of this function is protective in physiology and counteractive against aging-related disorders.

DOI 10.26508/Isa.202000917 | Received 25 September 2020 | Revised 4 October 2020 | Accepted 5 October 2020 | Published online 21 October 2020

\section{Introduction}

The nervous system plays a leading role in controlling whole-body physiology and pathophysiology, including aging-associated physiological declines. In mammals, different brain regions should have specific contributions to aging, for instance, the hippocampus has long been known for aging-related cognitive disorders, and we recently appreciated that the hypothalamus is important for the endocrine control of whole-body aging (Satoh et al, 2013; Zhang et al, 2013), although the underlying cell mechanism involves hypothalamic neural stem/progenitor cells (htNSC) (Zhang et al, 2017). Because protein or peptide secretion is a key feature of endocrine function, this project was designed to examine protein or peptide secretome of htNSC, and we found that these cells importantly secrete parathymosin (PTMS). PTMS is a protein which has been poorly understood except for its sequence and limited biochemical information in the literature. This protein was initially isolated from rat thymus and found to have $43 \%$ structural identity with thymosin, thus leading to this name (Haritos et al, 1985; Komiyama et al, 1986; Trompeter \& Söling, 1992), and later it was purified from liver and was also given a different name (Okamoto \& Isohashi, 2000). Based on its mRNA sequence, PTMS is highly conserved across species and is a small zinc-binding acidic protein containing 101-amino acid residues. Besides, PTMS was known to have an operative bipartite nuclear-localization signal which could interact with histone (Kondili et al, 1996; Trompeter et al, 1996). PTMS appears to be widely expressed in the body, much more than what the name suggested, and it was already clear that the thymus is not a key site for PTMS expression (Clinton et al, 1989; Okamoto \& Isohashi, 2000). On the other hand, very little is known about its function, and it is completely unknown if it is relevant to the brain, neurons, or hypothalamus. In this work, we discovered that PTMS is a brain-secretory protein which is transferable among neuronal nuclei, suggesting that brain is an endocrine organ through secretion and nuclear transfer of PTMS with a neuroprotective role in physiology.

\section{Results}

The secretome of htNSC inhibits cell senescence in a co-culture model

Cellular aging is characterized with replicative senescence by which cells cease to divide (Kuilman et al, 2010; López-Otín et al, 2013). To initiate the investigation of peptide and protein secretome of htNSC in affecting aging process, we started with a screening experiment to identify if there exists a peptide or protein released by htNSC to reduce senescence of primary fibroblasts. As shown in Fig 1A, coculture was set up using a transwell system with mouse skin fibroblasts (MSFs) in the lower compartment and htNSC spheres in the upper compartment, then we measured growth kinetics of fibroblasts at each passage. After 2 wk of cell passaging, growth of

${ }^{1}$ Department of Molecular Pharmacology, Albert Einstein College of Medicine, Bronx, NY, USA ${ }^{2}$ Institute for Aging Research, Albert Einstein College of Medicine, Bronx, NY, USA ${ }^{3}$ Diabetes Research Center, Albert Einstein College of Medicine, Bronx, NY, USA 


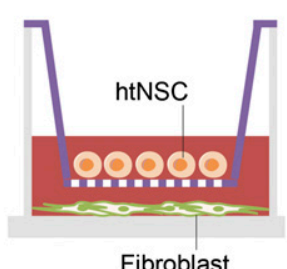

Fibroblast
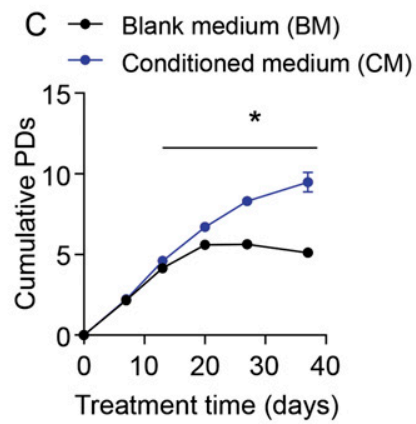

E
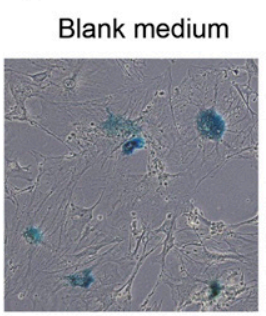

Conditioned medium

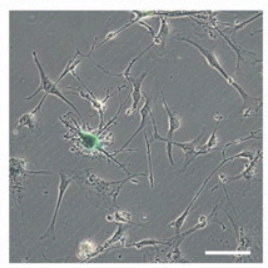

G

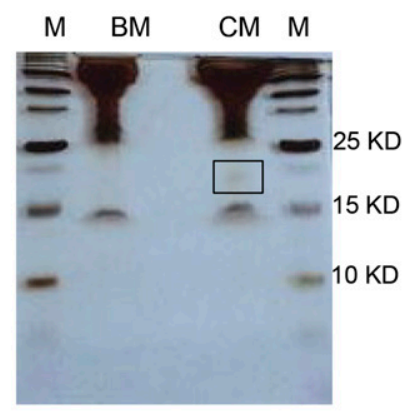

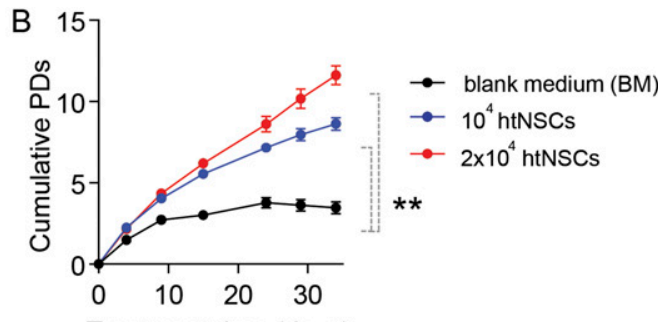

Treatment time (days)
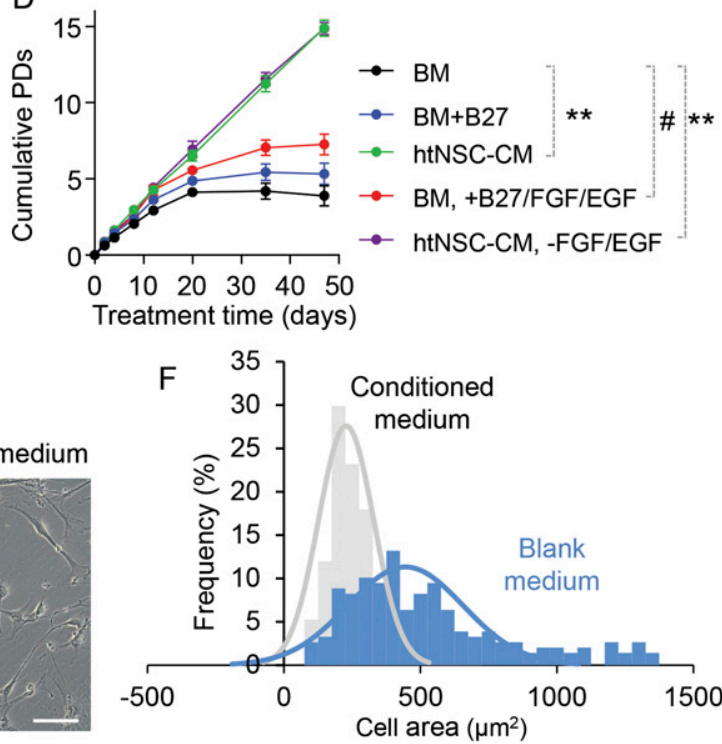

$\mathrm{H}$

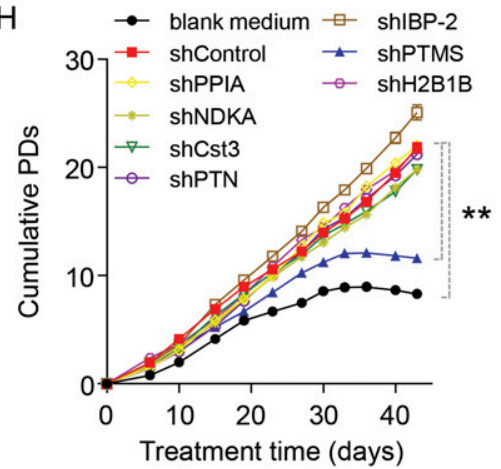

Figure 1. Identification of anti-senescence nuclear peptide PTMS released by htNSC.

(A) Schematic diagram depicting htNSC-primary fibroblast co-culture model. (B) Growth kinetics of primary mouse skin fibroblast co-cultured with indicated numbers of htNSC or with blank htNSC medium (labelled as "blank medium [BM]"). Cumulative Population Doublings (PDS) of fibroblasts were measured at each passage until the PD in BM group began to decrease. ${ }^{* *} P<0.01$ (ANOVA/post-hoc), compared between indicated groups across time points from day 9 to $35, n=3$ each group per time point. (C, D) Growth kinetics (PDs) of mouse skin fibroblasts co-cultured with BM or htNSC-conditioned medium (labelled as "CM") (C), or BM with B27, B27 plus bFGF, and EGF (10 ng/ml each) (D). ${ }^{*} P<0.05$ from day 13 to 37 , $\# P<0.05$ from day 35 to 47 , ${ }^{* *} P<0.01$ from day 20 to 47 , compared with time-matched groups indicated by solid lines (ANOVA/post hoc), $n=3$ each group per time point. (E, F) Senescence morphology of continuously passaged fibroblasts cultured with blank or htNSC-CM. (E, F) Cellular senescence displayed at passage 6 as revealed by $S A-\beta$-Gal staining (E) and cell size profiling (F). Scale bar, $100 \mu \mathrm{m}$. Data represented $n=134$ cells for blank group and $n=163$ for $C M$ group. (G) Representative image of silver-staining of SDS-PAGE separating peptides of htNSC-CM which showed anti-senescence effect, whereas BM was included to provide a baseline control. A smear area (indicated by black square) in the lane of htNSC-CM was analyzed by mass spectrometry for peptide identification. Molecular marker ladder (labelled as " $M$ ") was included on both sides. (H) Growth kinetics (PDs) of fibroblasts co-cultured with individual htNSC cell line expressing non-target shRNA control or specific shRNA targeting each of the mass spectrometry-identified peptides. Culture with BM was also included a background control, ${ }^{* *} P<0.01$ (ANOVA/post-hoc) compared between indicated groups across time points from day 15 to $43, n=4$ each group per time point. All values represent the mean \pm SEM fibroblasts reached a plateau stage as these cells stopped increasing the cumulative average population (Fig 1B), indicating that most cells became replicatively senescent. Co-culture with htNSC dramatically rescued the replicative senescence of fibroblasts in a dose-dependent manner, and a significant difference was observed after day 10 (Fig 1B). Then, to assess if this effect was mediated by any secreted factors, we obtained htNSC-conditioned medium and applied to fibroblasts. As the results showed in Fig 1C, htNSC-conditional medium greatly protected against replicative senescence of fibroblasts. Considering that the standard medium of htNSC culture contained additional ingredients (B27 supplements, EGF and bFGF) compared with the culture medium of fibroblasts, we further experimentally address this difference. Fibroblast replicative aging remained when these ingredients were added to its culture medium (Fig 1D), and on the other hand, the anti-aging effect of htNSC-conditioned medium remained intact even if EGF and bFGF were removed (Fig 1D). Besides proliferative kinetic, we analyzed senescence-related $\beta$-galactosidase (SA- $\beta$-gal) staining, cell morphology, and senescence-associated secretory phenotype. As shown in Fig $1 \mathrm{E}$ and $\mathrm{F}$, senescent fibroblasts were SA- $\beta$-gal positive, which became flattened and shape-enlarged, whereas co-cultured with htNSC significantly decreased SA- $\beta$-gal positive ratio and shifted the cell size frequency curve to the left. The mRNA of senescence-associated secretory phenotype cytokines of fibroblasts accumulated since passage 4, but it remained low at passage 10 when co-cultured with htNSC (Fig S1A). To summarize, htNSC secrete certain factor(s) to prevent against cellular replicative aging. 


\section{PTMS from htNSC secretome is anti-senescent}

In this context of the above findings based on co-culture and conditioned medium, we asked if any known neurotrophic factors could be accountable. We targeted a few prototypical ones, including brain-derived neurotrophic factor and glial cell line-derived neurotrophic factor (GDNF). In experiments, brain-derived neurotrophic factor and GDNF (10 ng/ml each) were added to fibroblast culture medium, but we found that they did not lead to any rescue of fibroblast replicative aging (Fig S1B). To unveil the potential antiaging peptides or proteins released by htNSC, we subjected htNSCconditioned medium to gel identification with silver staining and observed the presence of a smeared protein band between 15 and $20 \mathrm{kD}$ in htNSC-conditioned medium (Fig 1G). Thus, we isolated this area from the gel and analyzed the sequences of peptides in this area via nanoLC-MS/MS analysis. The most abundant candidate proteins or peptides revealed by this method included PPIA, NDKA, Cst3, PTN, IBP-2, Ube2v1, PTMS, H2B1B, and TI-225. Surprisingly, only Cst3, PTN, and IBP-2 were known as secretory factors, whereas others are either poorly characterized (such as PTMS and TI-225) or usually do not represent secretory proteins (such as PPIA, NADK, Ube2v1, and $\mathrm{H} 2 \mathrm{~B} 1 \mathrm{~B}$ ). Based on our recent finding which revealed that htNSC have an important function of abundantly releasing exosomes (Zhang et al, 2017), it is mostly likely that some of these proteins could be released through extracellular vesicles. We generated individual knockdown htNSC cell lines through lentivirus-harboring shRNA against each of these identified peptides or proteins. Through qRT-PCR, we confirmed that several htNSC lines developed from this strategy were comparable in terms of knockdown efficiency (Fig S2). We then co-cultured fibroblasts with each of these knockdown htNSC cell lines. The results revealed that PTMS knockdown dramatically abolished the antiaging effect of htNSC co-culture, whereas none of the other knockdowns did so (Fig $1 \mathrm{H}$ ). We thus analyzed the literature and recognized that PTMS has been poorly characterized except that it is known as a small zinc-binding acidic protein which is conserved across species, and it contains an operative bipartite nuclearlocalization signal and was found to interact with histone (Kondili et al, 1996; Trompeter et al, 1996). Hence, in the context of our results, we decided to study the possible endocrine feature of this protein.

\section{PTMS secretion from htNSC and its rapid nuclear transfer in vitro}

A suitable PTMS antibody should be necessary for studying its production and secretion in mouse models, although commercially there are a few PTMS antibodies against human species, we tested them for mouse samples but none of them turned out to be workable. Thus, we decided to generate a suitable antibody against this protein of mouse origin. We screened several versions of customized rabbit antibodies against different $\mathrm{N}$-terminal sequences of mouse PTMS and established an antibody, which was validated to be specific, as it specifically recognized an exogenously expressed PTMS but did not lead to a signal for various samples from ptms knockout mice (Fig S3). Using this antibody, we performed immunostaining for htNSC spheres and confirmed that htNSC indeed abundantly contained PTMS which largely co-localized with NSC markers Sox2 and nestin (Fig 2A). Furthermore, we profiled the kinetics of PTMS secretion by htNSC. As shown in Fig 2B, PTMS secretion from htNSC was quickly detectable within $2 \mathrm{~h}$ into serumfree medium and reached a steady state at $6 \mathrm{~h}$, indicating that PTMS secretion by htNSC is fast. We were interested in studying the property of PTMS release independent of its gene production, and thus we generated an in vitro htNSC model with exogenous expression of HA-tagged PTMS. Because the N-terminal could be important for mediating its release, we added HA tag to the C-terminal of PTMS protein. Various controls were designed, including the size- and sequence-matched control based on using scrambled cDNA sequence of PTMS, and HA was also tagged at the C-terminal. Indeed, both were expressed in htNSC according to HA staining, but notably PTMS-HA was present mainly in the nucleus, whereas Control-HA was also widely present in the cytoplasm (Fig 2C), which further suggested that PTMS can work in the nucleus. Also, through analyzing HA in culture medium, we confirmed that PTMS-HA was abundantly released, whereas control-HA did not lead to any level of release. Subsequently, we studied how PTMS from secretion could act on target cells, for example, whether it act on cell membrane as similarly as many other peptidyl hormones do, or if it could be transferred into cells because it might function in the nucleus. To answer this question, we obtained conditioned medium from htNSC expressing PTMS-HA versus Control-HA, and then added to the culture of various other type cells including hypothalamic neuronal line GT1-7, fibroblasts, hepatic cell line HepG2, and kidney cell line HEK293, as elucidated in Fig 2D. After $24 \mathrm{~h}$ of incubation, we used HA immunostaining to examine if HA tag could be present in these cells. The results showed that PTMS-HA was prominently transferred into all these cells, but Control-HA did not at all (Fig 2E). According to DAPI staining, HA-tagged PTMS entered and accumulated in the nuclei of these various cell types (Fig 2E). Because only some cells captured PTMS, it can suggest that a particular stage of cell cycling is needed for these cells to receive PTMS. Additional analysis showed that this nuclear transfer occurred very rapidly, as HA was detectable within 15 min in various recipient cells such as GT1-7 cells, HEK293 cells, and HepG2 cells (Fig $2 \mathrm{~F}-\mathrm{H}$ ). Hence, agreeing with the biochemical information that PTMS contains a nuclear-localization signal (Kondili et al, 1996; Trompeter et al, 1996), our data suggest that this secretory protein can travel and cross the plasma membrane, traffic through the cytoplasm, and enter the nucleus of various recipient cells.

\section{PTMS transfer from htNSC to neurons in the hypothalamus}

As revealed previously, htNSC are Sox2 positive, mostly located in the hypothalamic third ventricle wall and the MBH parenchyma ( $\mathrm{Li}$ et al, 2012, 2014; Zhang et al, 2017; Cai \& Khor, 2019; Tang et al, 2020; Xiao et al, 2020). Consistently, the cerebroventricular system has been increasingly recognized for the role of neuropeptide in physiological regulation (Noble et al, 2018). From the results of Sox2 and PTMS co-immunostaining of mouse brain sections, PTMS was present in the nucleus of all htNSC (Fig 3A). We confirmed that this PTMS antibody was suitable for immunostaining, as it did not generate any specific signals when brain sections from ptms knockout mice were used. Because the hippocampal dentate gyrus and the subventricular zone in mouse brain are also known to 

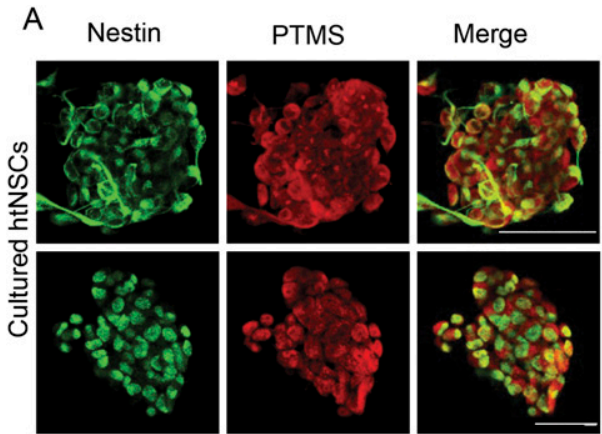

Sox2

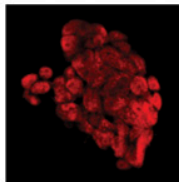

PTMS

Merge

C htNSC control-HA htNSC ${ }^{\text {PTMS-HA }}$
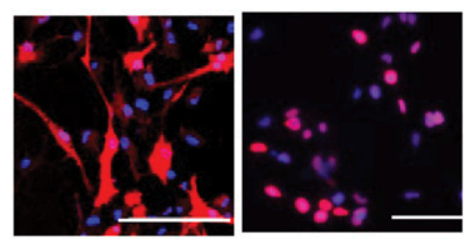

E

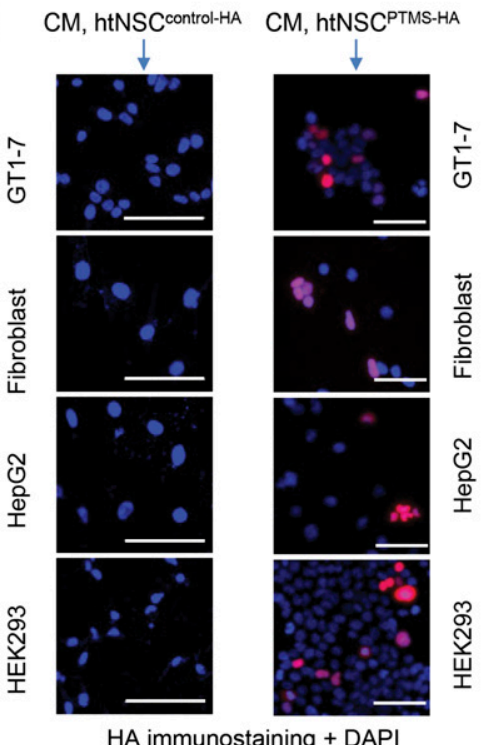

B PTMS —- - - - - - -

Loading

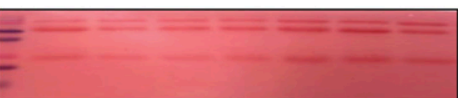

D

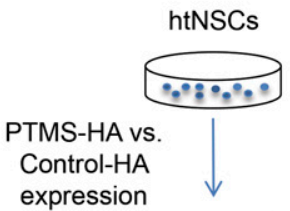

Donor htNSCs

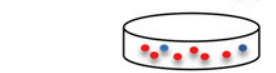

Conditioned medium (CM)

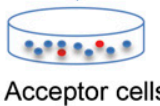

$\mathrm{F}$

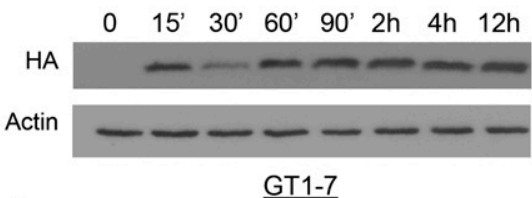

G

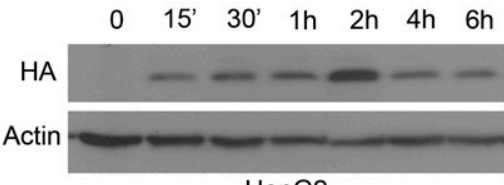

HepG2

$\mathrm{H}$

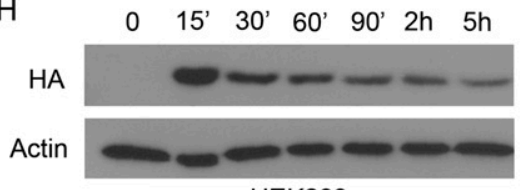

Figure 2. Protein expression, secretion and transfer of PTMS by htNSC in vitro.

(A) Representative sections of htNSC spheres were double immunostained for PTMS and neural stem cell marker Nestin or Sox2. Scale bar, $50 \mu \mathrm{m}$. (B) htNSCconditioned medium (CM) was collected in a time course of $72 \mathrm{~h}$ and was detected for PTMS levels by immunoprecipitation-Western blot. Ponceau staining was used as a loading control. (C) htNSC were infected with the lentiviruses expressing HA-tagged PTMS (PTMS-HA) or HA-tagged scramble control (Control-HA) driven by CMV promoter. (D, E, F, G, H) CM of donor htNSC expressing PTMS-HA (labelled as htNSC ${ }^{\text {PTMS-HA }}$ ) versus Control-HA (labelled as htNSC ${ }^{\text {control-HA }}$ ) was added to various acceptor cells for various of time, as elucidated in (D). (E) Subcellular localization of HA-tagged protein in acceptor cells was detected by HA immunostaining after $24 \mathrm{~h} \mathrm{CM}$ incubation (E). DAPI nuclear staining indicated all cells in the fields. Scale bar, $100 \mu \mathrm{m}$. (F, G, H) GT1-7 (F), HepG2 (G), and HEK293 (H) cells treated with CM of htNSC ${ }^{\text {PTMSI-HA }}$ were collected at the indicated time periods of incubation, thoroughly washed, and lysed for HA Western blot. Western blot did not reveal any HA signal in the above cells incubated with htNSC ${ }^{\text {control-HA }}$ CM.

HEK293

contain adult NSCS, we examined these cells but found that PTMS was absent in either of them (Fig 3A). This difference can suggest that there are substantial differences between htNSC and other NSC types. On the other hand, in addition to htNSC, PTMS protein was found positive in majority of neurons throughout the brain and particularly in the cortex and hippocampus (Fig 3B). Using in situ hybridization, we also confirmed that ptms mRNA was detectable in htNSC as well as neurons in various brain regions (Fig S4). It is possible that PTMS is expressed in non-neuronal cells such as astrocytes and microglia (which was not addressed in this study because of technical limitation), but based on morphology, neurons are clearly important for abundantly expressing PTMS. Because of the feature that PTMS is transferable among cells, an interesting question was if htNSC could donate PTMS to neurons in vivo. To answer this question, we generated a lentivirus expressing PTMSHA driven by Sox2 promoter, using a lentiviral promoter system which is effective for inducing gene expression specifically in Sox2positive cells which belong to the populations of NSCS and early offspring before terminal differentiation. This method has been established in the literature and our previous studies (Li et al, 2012, 2014; Zhang et al, 2017; Tang et al, 2020; Xiao et al, 2020), as we repeatedly confirmed that this Sox 2 promoter-driven lentivirus delivered an ectopic protein such as GFP specifically into Sox2positive cells without targeting neurons. We delivered these lentiviruses into the mediobasal hypothalamus (MBH) of mice; at 1 wk after injection, hypothalamic sections were made for examining the expression of the delivered gene. Indeed, lentiviral induction of PTMS-HA led to HA signals in Sox2-positive cells (mostly in the 3V wall in the $\mathrm{MBH}$, Fig $3 \mathrm{C}$ left). On the other hand, $\mathrm{HA}$ signals were also widely detected in many Sox2-negative cells (mostly in nuclei in $\mathrm{MBH}$ parenchyma, Fig $3 \mathrm{C}$ right); through neuronal HuC/D immunostaining, we confirmed that these cells were neurons. This 
A

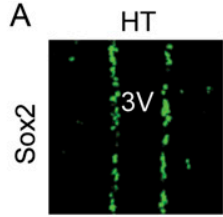

Hip (DG)
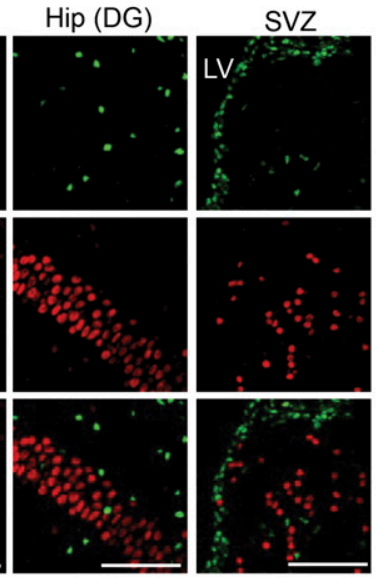

C

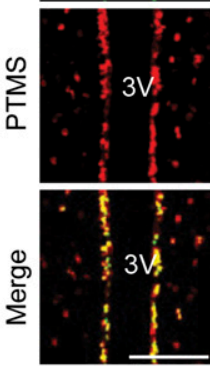
Lenti- $\mathrm{P}_{\text {Sox2 }} / \mathrm{PTMS}-\mathrm{HA}$ MBH injection
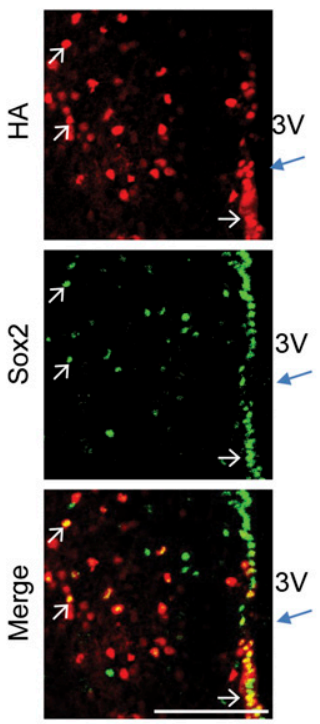

$\mathrm{MBH}$ with $3 \mathrm{~V}$
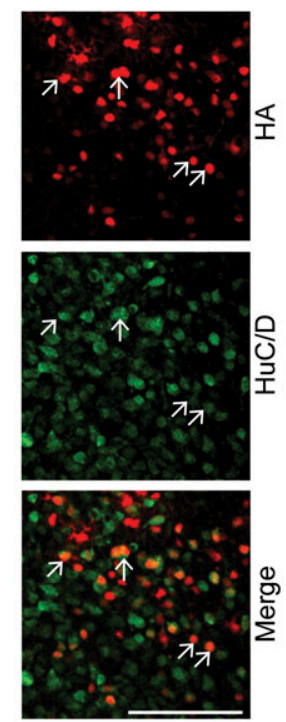

$\mathrm{MBH}$ parenchyma

$\frac{2}{2}$
B Hippocampus
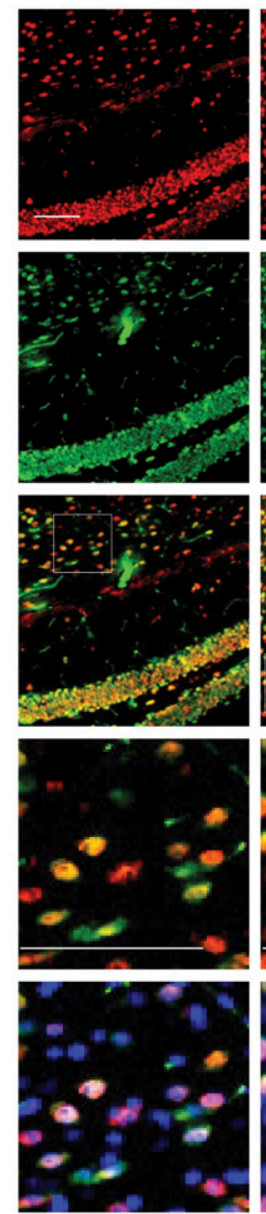
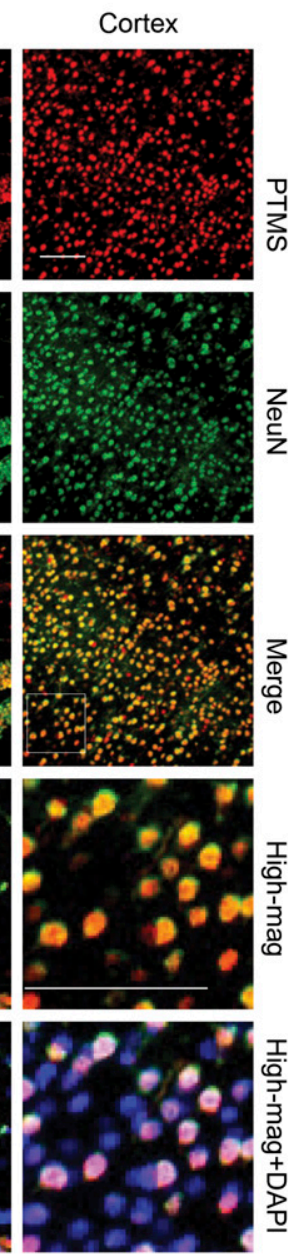

Figure 3. PTMS transferring from htNSC to neurons in the hypothalamus.

(A) Representative brain sections of 3-mo C57BL/6 mice were immunostained for PTMS and NSC marker Sox2, focusing on NSC-positive regions including the third ventricle (3V) region of the hypothalamus (HT), dentate gyrus of hippocampus (Hip), and sub-ventricle zone of forebrain (SVZ). DAPI nuclear staining was included. (B) Brain sections across the cortex or the hippocampus of 3-mo male C57BL/6 mouse were generated and co-immunostained for PTMS and neuronal marker NeuN. Representative regions of parietal cortex and hippocampal dentate gyrus are presented. High-magnification views (High-mag) of outlined regions in merge images were included to show more details. (C) 3-mo C57BL/6 mice received unilateral injection of Sox2 promoter-driven PTMSHA lentivirus in the mediobasal hypothalamus (MBH). Brain sections were made at day 7 postinjection and immunostained with Sox2 and neuronal marker HuC/D. 3V region of $\mathrm{MBH}$ containing Sox2-positive cells and $\mathrm{MBH}$ parenchyma containing HuC/D-positive neurons are presented in left and right panels, respectively. Small arrows pointed to co-localization of HA with Sox2 (left panel) or HuC/D (right panel). Scale bar, $100 \mu \mathrm{m}$ observation was consistent with the in vitro data presented in Fig 2, suggesting that PTMS is not only secretory by htNSC but transferable from htNSC to neurons in the hypothalamus.

\section{Contribution of the hypothalamus to PTMS in the CSF}

We then measured PTMS levels in the cerebrospinal fluid (CSF) of mice, and its blood level was measured for a comparison. As shown in Fig 4A, PTMS concentration in the CSF is five times higher than that in the blood serum. In this context, we asked if hypothalamic PTMS release especially through htNSC could be important for its level in the CSF. In our previous research (Zhang et al, 2017), we have appreciated that lentiviruses when injected into the hypothalamic dorsal third ventricle infect only the cells on the surface but barely penetrated into the parenchyma or travel to other brain regions. To address this question, we performed an experiment by injecting lentiviruses of ptms shRNA versus scramble control into the hypothalamic third ventricle of mice, resulting in a substantial knockdown of PTMS expression in htNSC on the third ventricle surface compared with the control group (Fig 4B). Importantly, we found that this knockdown led to $\sim 40 \%$ drop of PTMS concentration in the cerebrospinal fluid (Fig 4C). Hence, although htNSC in the brain is rather a small population, it plays an important role for maintaining PTMS level in the cerebrospinal fluid. For comparison, a cortex injection of lentiviruses was performed in which PTMS-HA versus Control-HA were controlled by CMV promoter. Indeed, the hypothalamic third-ventricle injection led to HA expression specifically in Sox2-positive htNSC on the surface of third-ventricle wall in both groups, as represented by PTMS-HA group in Fig 5A and Control-HA group in Fig S5A. Injection of these PTMS-HA and Control-HA lentiviruses in the cortex is presented in Figs $5 B$ and $\mathrm{S} 5 \mathrm{~B}$, respectively. Using qRT-PCR primers which recognized only exogenous ptms-HA, we confirmed that ptms-HA mRNA was induced specifically in the hypothalamus or the cortex (Fig 5C). We obtained both CSF samples and brain sections from these mice for HA Western blot and immunostaining. As shown in Fig 5D, the hypothalamic injection of PTMS-HA led to an evident induction of 
A
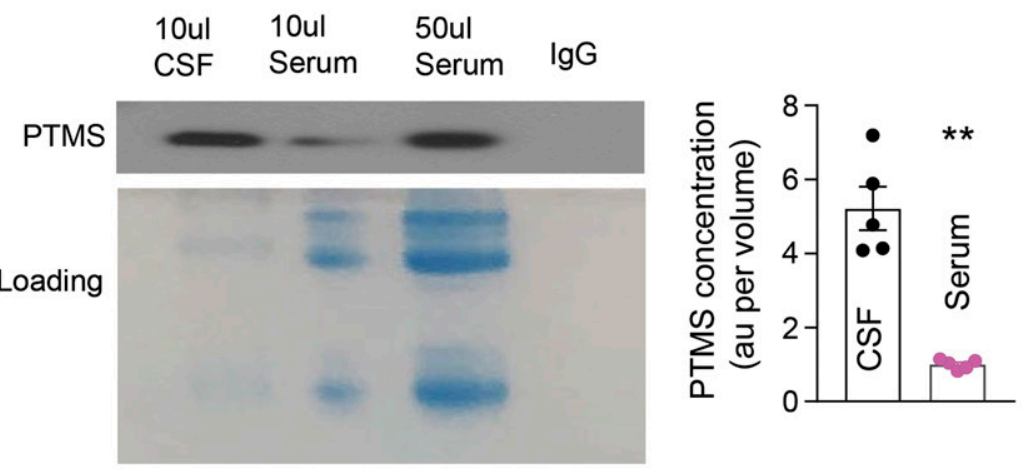

B

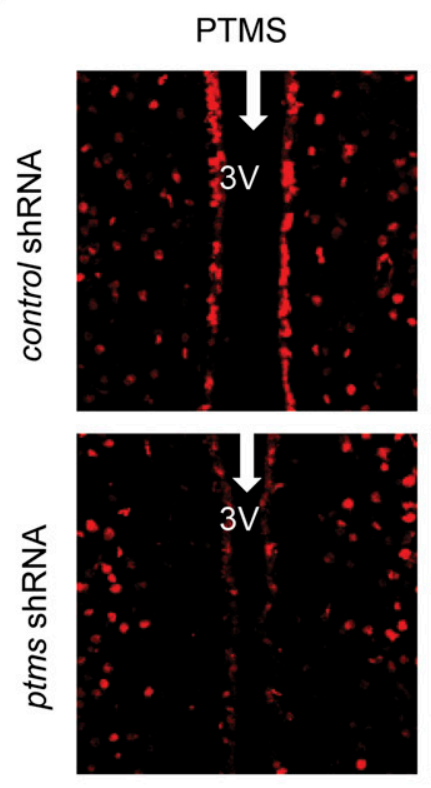

C

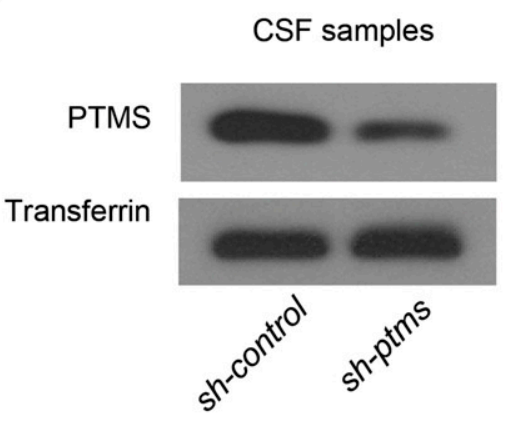

Sox2
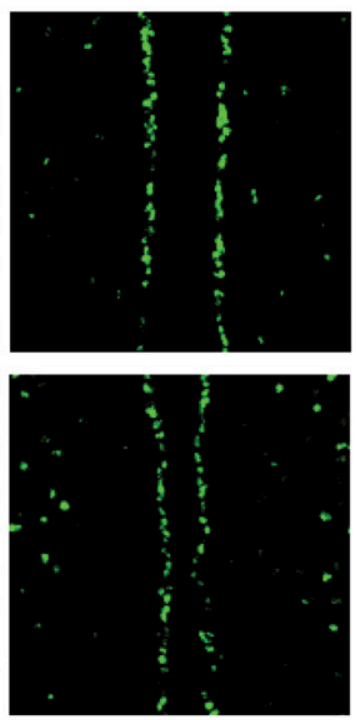

Merge
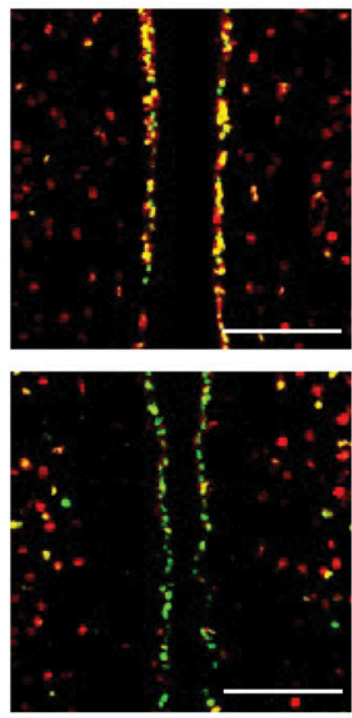

Figure 4. Contribution of the hypothalamus to PTMS in the CSF.

(A) PTMS levels in blood serum versus CSF were detected by immunoprecipitation (IP)-Western blot (left panel), and the band intensities (au, arbitrary unit) were quantified (right panel). Matched IgG was used as a negative control of IP procedure, and Commassie staining was used as a loading control. (B, C) Injection of ptms-shRNA (sh-ptms) or scramble control shRNA (sh-control) lentiviruses into hypothalamic third ventricle of 3-mo male C57BL/ 6 mice (B), and at 1 mo postinjection, PTMS levels in CSF were measured by Western blot (left panel, transferrin as a loading control) and quantified (right panel). Scale bar, 100 $\mu \mathrm{m} .(\mathbf{A}, \mathbf{C}){ }^{*} \mathrm{P}<0.05$ (two-tailed unpaired $t$ test), $\mathrm{n}=4$ mice $(A)$ or 3 mice $(C)$ per group; values represent the mean \pm SEM.

this protein in the cerebrospinal fluid, but this effect was much weaker when cortex injection was performed. HA immunostaining showed that hypothalamic injection of PTMS-HA led to more appreciable numbers of $\mathrm{HA}$-positive cells in other brain regions than did by cortex injection (Fig 5A and B), whereas Control-HA was absent in any of these brain regions (Fig S5A and B). Through Western blot, we were still able to detect a level of PTMS-HA in a few other brain regions such as the thalamus (Fig 5E), suggesting that PTMS from the cortex was transferable although to a limited extent.
Taken together, PTMS from the brain and particularly the hypothalamus provides an important contrition to its level in the CSF.

\section{PTMS transfer among neurons in different brain regions}

Because we observed that neurons also produce PTMS, a relevant question was, if neurons could act as not only recipients but also donors of PTMS? We have obtained some initial information from cortex injection in Fig 6, but because these lentiviruses were not neuron specific, we 
A $3 V$ injection of lentiviral PTMS-HA
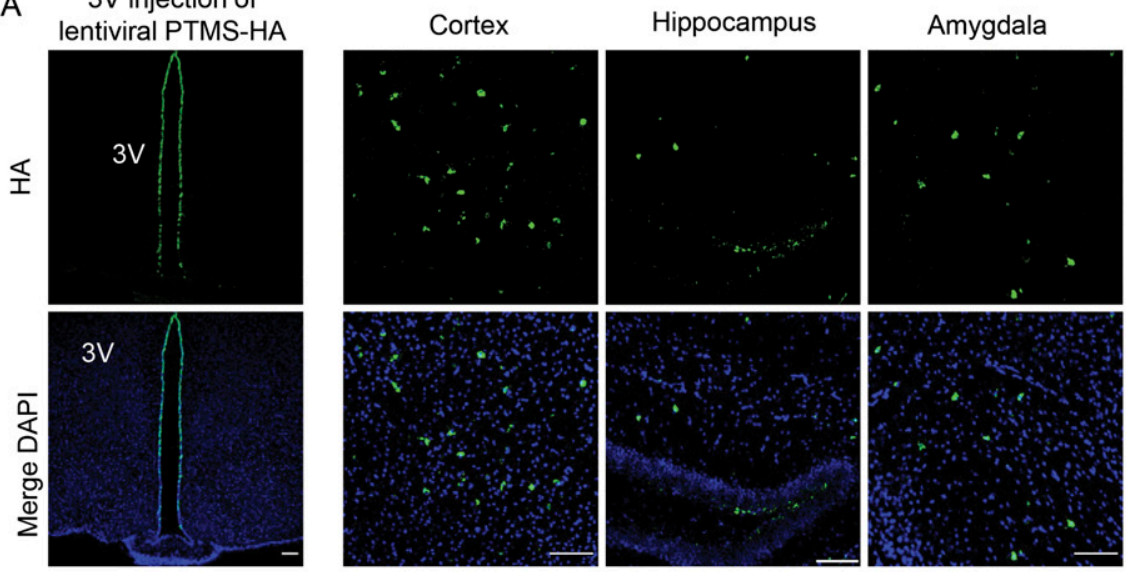

B Cortex injection of lentiviral PTMS-HA
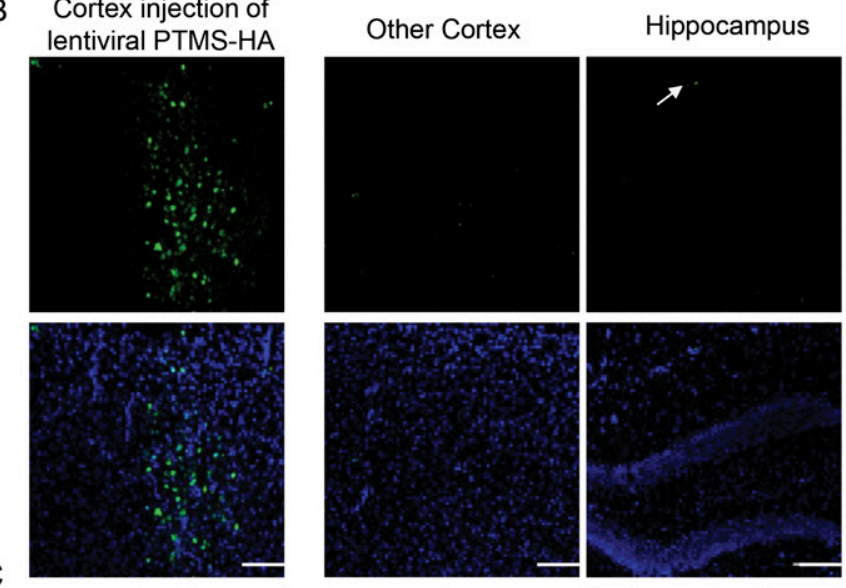

Thalamus
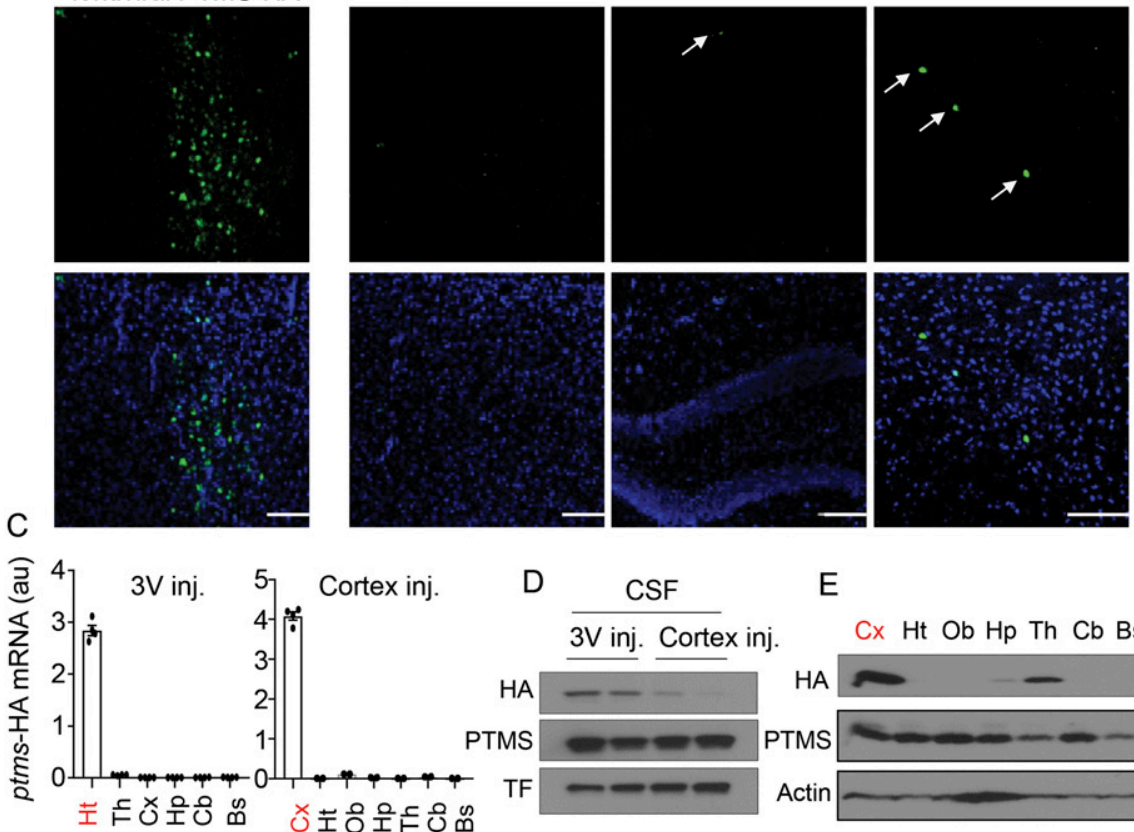

E

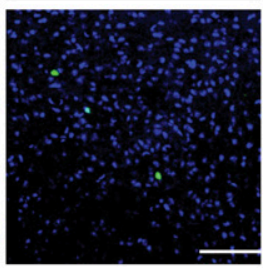

$\mathrm{Cx} \mathrm{Ht} \mathrm{Ob} \mathrm{Hp}$ Th $\mathrm{Cb}$ Bs
Figure 5. Transfer of hypothalamus and cortex PTMS to other brain regions.

4-wk male C57BL/6 mice received hypothalamic third ventricle or cortex injection of CMV-promoter driven PTMS-HA versus Control-HA, and at 2 wk postinjection, brain and cerebrospinal fluid (CSF) samples were collected for following analyses. (A, B) Induction of PTMS-HA in hypothalamic third ventricle wall (A) and parietal cortex (B) as revealed via HA immunostaining. Small arrows pointed to some HA positive cells in transfer brain regions. Scale bar, 100 $\mu \mathrm{m}$. (C) Measurement of exogenous HA-tagged ptms mRNA by qRT-PCR for the injection brain region and other brain regions. (D) Measurement of PTMS in the CSF by HA and PTMS Western blots. Transferrin (TF) was used as a loading control. (E) Multiple brain areas of cortex injection group were examined for PTMS-HA expression across various brain areas. Scale bar, 100 $\mu \mathrm{m}$. Ht, hypothalamus; Hp, hippocampus; Th, thalamus; $\mathrm{Cx}$, cortex; $\mathrm{Cb}$, cerebellum; Bs, brain stem. Additional information of control lentivirus injections are presented in Fig S5. $\mathrm{n}=4$ mice (C) per group; values represent the mean $\pm \mathrm{SEM}$. decided to use a neuron-specific lentivirus for independent experiments. Therefore, PTMS-HA and Control-HA lentiviruses were made under neuronal synapsin promoter and were injected into the hypothalamus versus hippocampus, as represented in Figs 6 and S6. We found that hypothalamic and hippocampal neurons were both able to transfer PTMS into a few different brain regions (Fig 6A and B). PTMS transfer from hypothalamic neurons was generally stronger than that from hippocampal neurons, agreeing with the classical endocrine role of the hypothalamus. In summary, consistent with being a special type of hypothalamic endocrine cells, htNSC are important for endocrine release of PTMS, whereas brain neurons seem to have a secondary contribution to this process.

\section{PTMS transfer from the brain to the blood}

Considering this gradient between the CSF and blood, we asked if brain PTMS could provide a contribution to blood PTMS. To answer this question, we used lentiviral injection to overexpress PTMS-HA in both the hippocampus and hypothalamus of mice and then examined if this HA-tagged peptide could be detected in the blood of these animals. Using qRT-PCR primers which specifically detected exogenous tagged ptms, we confirmed that injection of lentiviral PTMS-HA increased the mRNA levels of exogenous HAtagged ptms only in the brain without any leakage into the peripheral tissues. HA-tagged PTMS was readily detectable in the CSF through HA immunoblotting (Fig $6 \mathrm{C}$ ). This resulted in $\sim 60 \%$ increase in PTMS level in the CSF (Fig 6C). Thus, agreeing with the fact that the size of neuronal population is much larger than that of htNSC, the total contribution of neuronal PTMS to the CSF is substantial. Excitingly, we observed that HA-tagged PTMS was present in the blood of these mice (Fig 6D), which led to 25\% increase in blood PTMS level compared with the control group (Fig 6D). Hence, because PTMS has been known to be produced and released by peripheral organs such 
A Unilateral hypothalamus injection
with Lenti- $P_{\text {syn }} /$ PTMS-HA
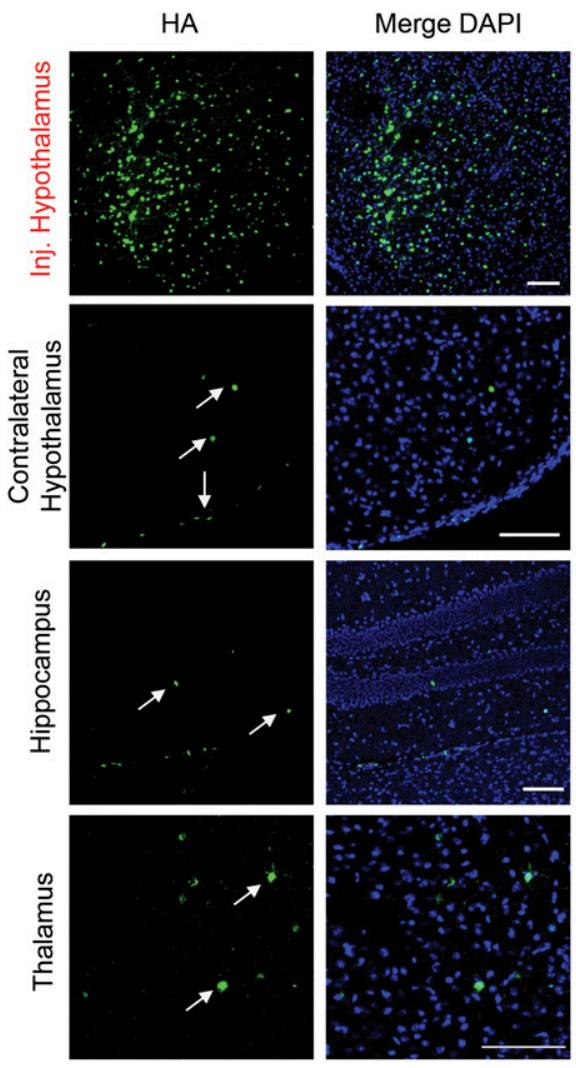

B
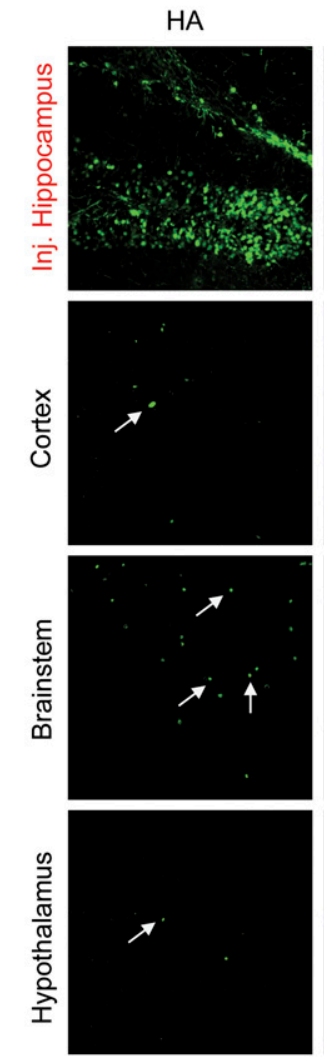

D

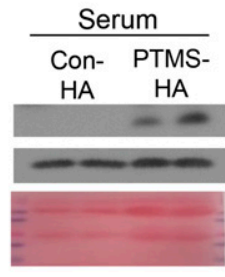

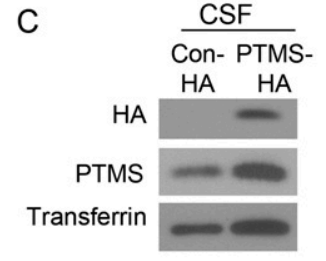

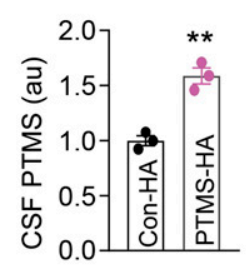

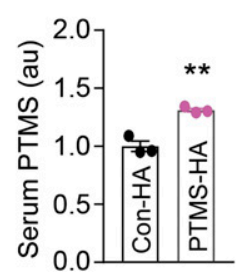
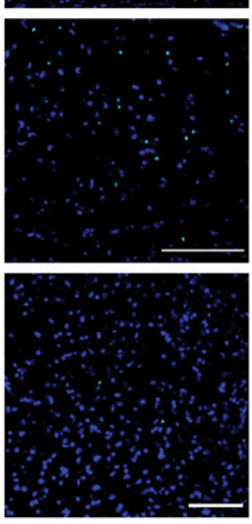

の
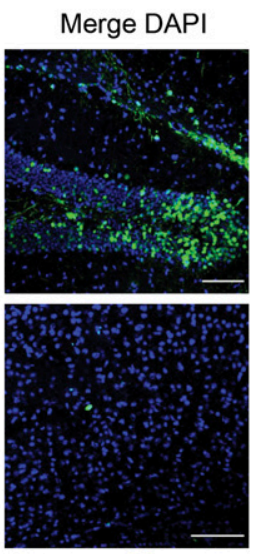

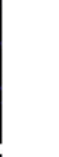

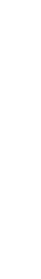

Figure 6. Transfer of hippocampal/hypothalamic PTMS to other brain regions and the blood.

(A, B) 3-mo male C57BL/6 mice were injected with a dose of synapsin promoter-driven PTMS-HA lentivirus in one side of the posterior hypothalamus (A) or both sides of the hippocampus targeting CA1 and dentate gyrus (B). At 2 wk post injection, brain sections were immunostained for $\mathrm{HA}$ in the injected regions versus other brain areas. Small arrows pointed to some HA positive cells in transfer brain regions. Scale bar, 100 $\mu \mathrm{m}$. Additional information of control lentivirus injections are presented in Fig S6. (C, D) 3-mo male C57BL/ 6 mice received bilateral injections of neuronspecific lentivirus of synapsin promoter-driven PTMS-HA (labelled as "PTMS-HA") versus Control-HA (labelled as "Con-HA") in the hippocampus (targeting CA1 and dentate gyrus) and posterior hypothalamus, and at 2-wk post injection, secretion of PTMS-HA to the CSF (C) and the serum (D) was assessed via IP-Western blotting. (C, D) Transferrin blot (C) or Ponceau S staining (D) was used as loading control. ${ }^{* *} P<0.01$ (two-tailed unpaired $t$ test), $n=3$ mice per group; values represent the mean \pm SEM.

\section{represent the mean \pm SEM.}


A

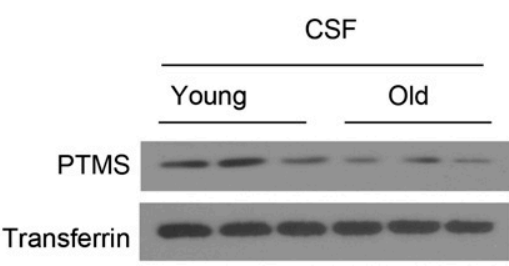

C
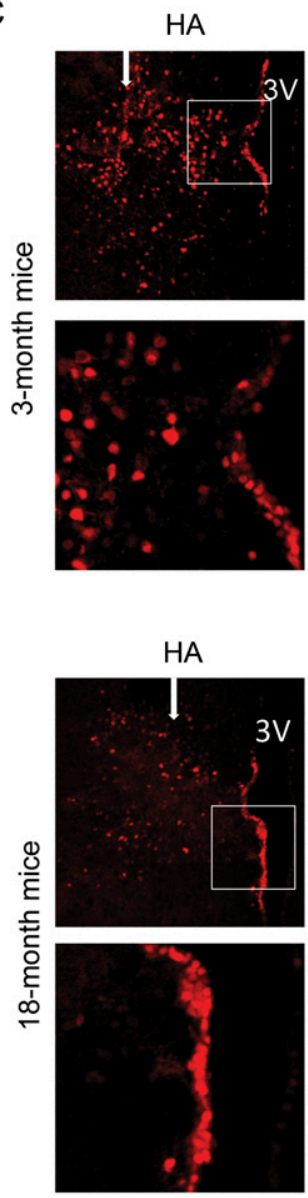

B

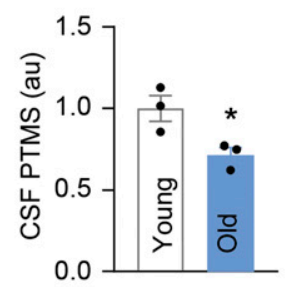

Figure 7. PTMS secretion declines during aging. (A, B) PTMS levels in the CSF of male C57BL/ 6 mice at young ( $4 \mathrm{mo})$ or old ( $24 \mathrm{mo})$ age were measured by Western blot (A) which were quantified in panel (B). Transferrin was used to as a loading control. (C) Young (3-mo) or middle aged (18-mo) male C57BL/6 mice received unilateral injection of the same dose of Sox2 promoter-driven PTMS-HA lentivirus in the mediobasal hypothalamus. White arrows point to the site of injections. At 1 wk postinjection, hypothalamic sections were generated for HA immunostaining to show the levels of both primary expression of PTMS-HA in Sox2-positive cells and secondary transfer of PTMSHA into Sox2-negative cells. Sox2 co-immunostaining was performed to reveal Sox2-positive cells in this region. Lower panels in show high-magnification views of outlined areas. Scale bar, $100 \mu \mathrm{m}$. 3V: hypothalamic third ventricle. ${ }^{*} P<0.05$, two-tailed unpaired $t$ test $(B), n=3$ mice per group; values represent the mean \pm SEM.

with young htNSC, aged htNSC are dysfunctional in producing, secreting, or transferring PTMS. Hence, physiological aging is associated with a dramatic decrease in PTMS secretion by htNSC.

\section{PTMS loss in the hypothalamus versus hippocampus in affecting aging behavior}

Because the brain is responsible not only for PTMS level in the CSF but also has a contribution to the blood PTMS level, we further evaluated the prediction that the brain or certain brain regions should have a critical role for anti-aging effects of PTMS. To do so, we first employed several knockdown mouse models via bilateral micro-injection of ptms shRNA lentiviruses into different brain regions of middle-aged male C57BL/ 6 mice. As elucidated in Fig 8A, same dose of ptms shRNA versus control scramble shRNA lentiviruses were bilaterally injected to each of these brain regions using the same cohort of age- and conditionsmatched mice. After injections, these mice were longitudinally monitored for aging-related behavioral dysfunctions through a batter of noninvasive behavioral assays, including Y-Maze, grip test, open field test, novel object exploration, cognition, sociality, and nest building tests. No evident differences were found in either knockdown group compared with their matched controls at 2 mo after injection. However, at $5 \sim 6$ mo after injection, we found that hypothalamic ptms knockdown mice performed much worse than the controls across almost all these behavioral tests (Fig 8B). Hippocampal ptms knockdown mice performed worse in a few hippocampus-dependent behaviors including Y-maze and social recognition but did not affect other behavioral functions compared with the controls (Fig 8C). Although there could be many additional experiments to compare other aspects of aging, this behavioral difference represented a piece of valuable information to suggest 
A

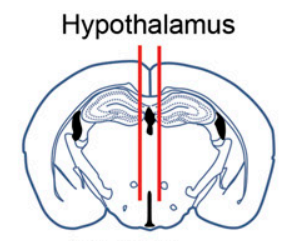

$-1.70 \mathrm{~mm}$

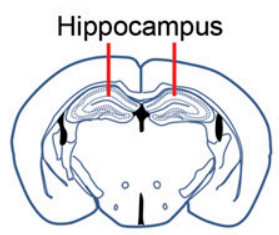

$-1.80 \mathrm{~mm}$

B

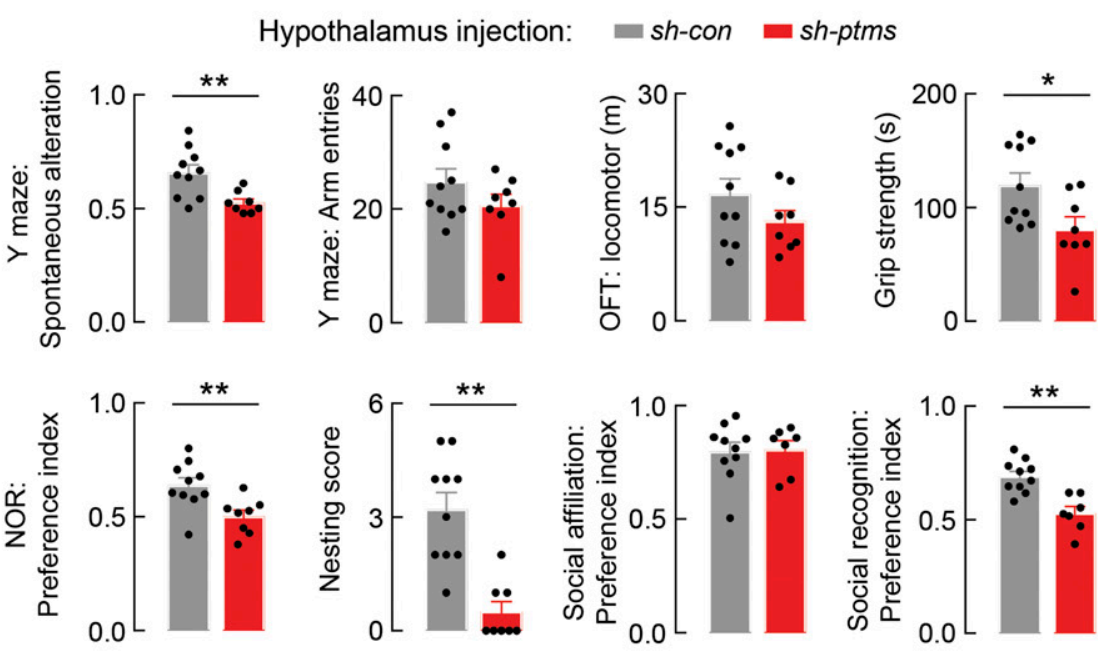

C

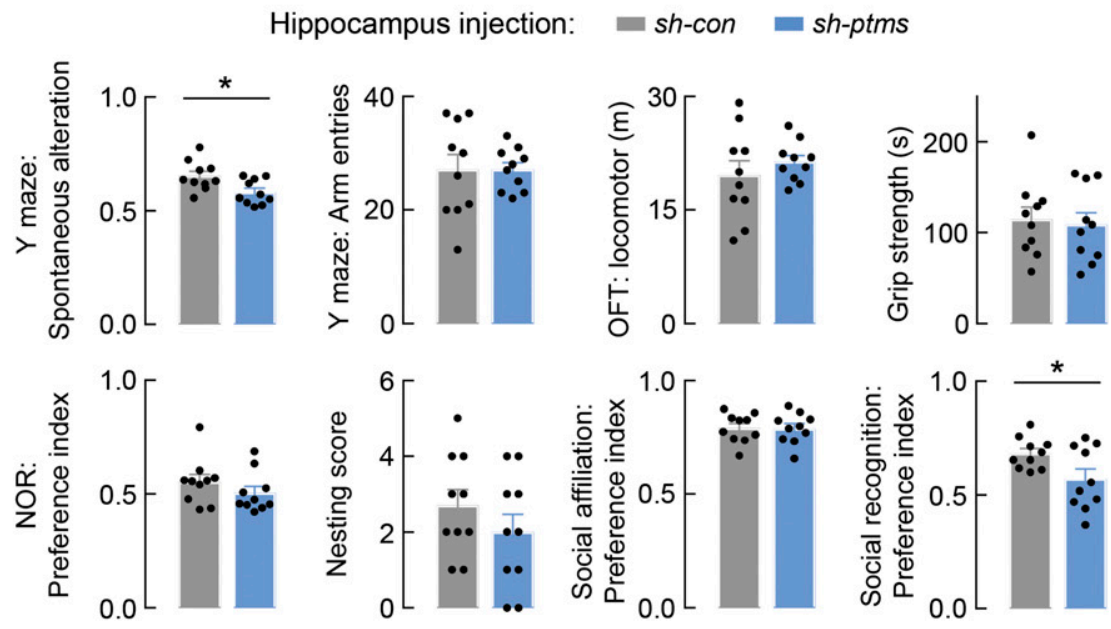

Figure 8. PTMS loss in the hypothalamus or hippocampus leads to aging-related behaviors. Middle-aged male C57BL/ 6 mice ( 15 mo old) were bilaterally injected with lentiviruses of scramble control shRNA (labelled as "sh-con") or ptms shRNA (labelled as "sh-ptms") in the hypothalamus, hippocampus. (A) Injections targeted posterior hypothalamus, CA1 and dentate gyrus of hippocampus (bregma distance in each injection is labelled), as elucidated in (A). (B, C) Behavioral tests were performed at $5 \sim 6$ mo post viral injection (B, hypothalamus injection; $C$, hippocampus injection). ${ }^{*} P<0.05,{ }^{* *} P<0.01$, two-tailed unpaired $t$ test, $n=7-10$ mice per group; values represent the mean \pm SEM. that loss of PTMS in the brain provides a contribution to aging physiology, and such a functional loss in the hypothalamus is relatively more important which agrees with the role of hypothalamus in endocrine control of aging.

\section{Discussion}

Recently, the hypothalamus was found to play a role in health and lifespan control (Satoh et al, 2013; Zhang et al, 2013), and htNSC was shown to be responsible at least through the secretion of miRNAcontaining exosomes (Zhang et al, 2017). This previous knowledge suggested that htNSC have special endocrine functions in influencing aging physiology, but the endocrine secretion portfolio of these cells remained to be mapped. In this study, we performed protein screening using an in vitro co-culture model and found that htNSC secrete PTMS to prevent against cell senescence of fibroblasts in the co-culture system. Biochemically, PTMS is conserved across species and could work in the nucleus according to its sequence and some biochemical assay (Kondili et al, 1996; Trompeter et al, 1996); however, the biological significance of this protein is barely known. Here, we discovered that PTMS is evidently released by htNSC and is quickly transferred into the nuclei of other cells and particularly neurons in various brain regions. These findings further support that htNSC have special endocrine functions more than neurogenesis as its name suggested, a point which we began to 
elucidate in our previous studies (Zhang et al, 2017; Cai \& Khor, 2019; Tang et al, 2020).

Interestingly, neurons in various brain regions not only receive PTMS but also release PTMS and thus contribute to its levels in the CSF. Compared with various brain regions, the hypothalamus is relatively stronger in inducing PTMS transfer over a long distance, for example, from the hypothalamus to the cortex. This advantage of the hypothalamus is likely attributed to htNSC which do not exist in other brain regions. On the other hand, because the size of neuronal populations throughout the brain is much larger than that of htNSC, the total release of PTMS from neurons is immense, which can lead to a contribution to the blood level of PTMS. Thus, our study indicates that neuroendocrinology should not limited to a few small brain regions such as the hypothalamus but can be expanded to the majority (if not all) of neurons in the brain although they have been classically known for inducing synaptic transmission. However, the brain and the hypothalamus within the brain should represent different orders in performing endocrine functions. We propose a conceptual model that whereas the hypothalamus is a primary-order neuroendocrine organ, the rest of the brain is a second-order neuroendocrine organ which is influenced by the hypothalamus but can independently perform an endocrine function.

It is still unclear how PTMS works in the nuclei of different cells, but likely it might provide an effect through stabilizing some nuclear and chromatin structures, given that it was previously shown to bind to histone (Kondili et al, 1996). It also remains to be determined if a carrier should be needed for PTMS release and transfer, especially considering that PTMS is an acidic protein which logically might not be circulated and transferred freely by itself. We have obtained some results showing that extracellular vesicles (exosomes and microvesicles) of htNSC contain PTMS; however, to study this possibility is a different subject, and besides, we also recognize that extracellular vesicles might not be the only way involved.

Physiologically, we generated mouse models with ptms knockdown specifically in the hypothalamus versus hippocampus and the results indicated that PTMS is neuroprotective while the hypothalamus played a more overall role in supporting neurobehavioral functions. These studies, albeit limitedly, provided initial proof-of-concept evidence to suggest that PTMS secretion and transfer are physiologically important, whereas much more studies are needed to demonstrate the details. We also predict that PTMS secretion and transfer are important not only for the brain but also for peripheral tissues, but it remains to be studied if brain versus peripheral secretion of PTMS might cooperatively work to regulate whole-body physiology, and if the hypothalamus-brain orchestration might provide a regulatory role in this process.

\section{Materials and Methods}

\section{Cell culture}

Primary culture of htNSC was performed as described previously (Li et al, 2012; Zhang et al, 2017; Tang et al, 2020). In brief, the hypothalamus was dissected from newborn C57BL/ 6 mice, cut into small pieces of $\sim 1 \mathrm{~mm}^{3}$, and followed by digestion using TrypLE Express enzyme (Life Technologies) for $10 \mathrm{~min}$ at $37^{\circ} \mathrm{C}$. After centrifugation, cells were suspended in NSC medium composed of neurobasal-A (Life Technologies), 0.25\% GlutaMAX supplement (Life Technologies), 2\% B27 without vitamin A (Life Technologies), $10 \mathrm{ng}$ $\mathrm{ml}^{-1}$ EGF (Sigma-Aldrich), $10 \mathrm{ng} \mathrm{ml}^{-1}$ bFGF (Life Technologies), and $1 \%$ penicillin-streptomycin and seeded in ultralow-adhesion sixwell plates (Corning). 1 wk later, neurospheres were collected by centrifugation and trypsinized with TrypLE Express enzyme into single cells, passaged, and maintained in neurosphere culture until experimental use. MSFs were isolated from mice at postnatal day 7-14 with the enzymatic method as described (Rittie \& Fisher, 2005). Briefly, the mice were euthanized, and shaved, washed, and sterilized with $70 \%$ EtOH and thoroughly rinsed with PBS. A longitudinal dorsal incision was made on the skin from the tail to the snout, followed by incision around the truncus and whole skin removal. Several skins were placed with dermis-side down and floated onto $0.25 \%$ trypsin solution for overnight incubation at $4^{\circ} \mathrm{C}$. The dermis was peeled off from the epidermis with forceps, minced into small pieces $(<1 \mathrm{~mm})$ if necessary, and collected into DMEM medium with $10 \%$ FBS (growth medium). The dermis was then vigorously vortexed for 20-30 s and filtrated through a 10- $\mu \mathrm{M}$ nylon cell strainer (Falcon), and the recovered cells from the filtrate were plated onto cell culture dishes in growth medium and passaged every $3 \mathrm{~d}$ with $0.25 \%$ trypsin. HEK293T cells (CRL-3216), HEK293 (CRL-1673), and HepG2 (HB-8065) were purchased from ATCC, and GT1-7 cells were established as previously reported (Zhang et al, 2013), those cells were cultured in DMEM containing $10 \%$ fetal bovine serum and $1 \%$ penicillin-streptomycin. All cell lines used in this study were tested free of microbial (including mycoplasma) contamination and their morphology and growth characteristics were compared with published information to ensure their authenticity. Conditioned medium for the proteomic analysis and for the fibroblast treatment were generated in the same way.

\section{htNSC and fibroblast co-culture}

On the day before co-culturing, htNSC were passaged in ultra-low attachment dishes to form neurospheres in neural basal A medium plus B27 without antioxidants, and $10^{4}$ MSFs were seeded on the bottom of 12-well transwell (\#3460; Corning). On the day of coculturing, neurospheres or blank medium control were transferred to the insert of transwell (upper compartment) and were replaced with fresh neurospheres or blank medium control every 2 or $3 \mathrm{~d}$. Neurospheres always contained the indicated numbers of htNSC based on cell counting of aliquots. MSF at the bottom were trypsinized and counted when the confluency reached $80-90 \%$, and $10^{4}$ cells were replated. MSF were exposed to the same co-culturing conditions at each passage. These MSF grew similarly in the neurophere medium compared with standard fibroblast medium. The population doubling number of MSFs was calculated using the forsmula $\triangle \mathrm{PDL}=\log \left(\mathrm{n}_{\mathrm{f}} / \mathrm{n}_{0}\right) /$ $\log _{2}$, where $N_{0}$ is the initial number of cells that were seeded and $n_{f}$ is the final number of cells that were harvested.

\section{Stable shRNA-expressing htNSC lines}

Using the method as previously described (Li et al, 2012; Zhang et al, 2017), various shRNA lentiviruses-infected htNSC were selected with 
puromycin at $1 \mu \mathrm{g} / \mathrm{ml}$ for $7-10 \mathrm{~d}$, and then maintained in regular culture medium supplemented with $0.5 \mu \mathrm{g} / \mathrm{ml}$ puromycin for maintenance.

\section{Plasmids and recombinant lentiviruses}

Various versions of lentiviral shRNA vectors and controls were purchased from Sigma-Aldrich, including PTMS shRNA (TRCN0000346743), PPIA/CYPA shRNA (TRCN0000101190), NDKA shRNA (TRCN0000360520), Cystatin C shRNA (TRCN0000055249), Pleiotrophin shRNA (TRCN0000071676), Hist1h2bb shRNA (TRCN0000093066), IGFBP-2 shRNA (TRCN0000012859), and Ube2V1 shRNA (TRCN0000039509). The plasmid plenti-CMV-ptms-HA was obtained by inserting coding sequence of ptms-HA into the plenti6 backbone (Invitrogen) with $\mathrm{BamHI}$ and Xhol sites. HA was tagged at the C-terminal of PTMS to protect its $\mathrm{N}$-terminal which involves in mediating its secretion. Size-matched control sequence was based on scrambled version of ptms which was synthesized and verified by Genewiz, and was inserted into the plenti6 backbone to generate plenti-CMV-controlHA. The scrambled control sequence of ptms: 5-GGATCCGCCACCATGCGCGAAGAAAAAGAAGATGAAAAAGATGAAGATGCGATGCTGAAACC GGAAGATAACGGCGAAGAAAAAGAAGCGGATGAAGTGGAAGGCGATACCGC GAAAGAAGATGAAACCCTGGCGGAAGCGGAACGCAGCGAAAAAGAAAAAGA AGAAGGCGCGGCGGAAGAAGCGGATGAACGCGAAAAAGAAAAGAAGAAA GCGAACGCACCGTGGATGAAGAAAACGAAAAAGAACAGAAAGGCAGCCCGG AAAGCAAAGCGAAAGAAGCGGGCGATGCGCGCGTGGTGGGCGTGGAAGAA GGAAGCGGATACCCATACGATGTTCCAGATTACGCTTGACTCGAG-3. The plasmid pSox2-ptms-HA and the scrambled control were obtained by replacing the Cre with ptms or its scrambled control sequence in the lentiviral plasmid pSox2-Cre which was described previously ( $\mathrm{Li}$ et al, 2012; Zhang et al, 2017). Synapsin promoter-directed lentiviral vector were obtained by inserting ptms or its scrambled control sequence into the Synapsin lentiviral plasmid with BamHI and Pcil sites. Lentiviruses were produced by transfecting HEK293T cells with corresponding viral and packaging plasmids, purified by ultracentrifugation and titrated using the p24 ELISA kit as described previously (Zhang et al, 2017).

\section{Cell senescence, size and secretome assays}

\section{Cell senescence analysis}

Senescence in cell culture was determined by Senescence $\beta$-Galactosidase (SA- $\beta$-Gal) Staining Kit (\#9860; Cell Signaling) according to the manufacturer's instruction as previously described (Tang et al, 2013). The developed blue color on cells was captured with the Zeiss Axiocam HRc camera of Zeiss Observer at the imaging core facility at the Albert Einstein College of Medicine.

\section{Cell size profiling}

Cells were trypsinized into single cells and mounted on a hemocytometer, and cell images were captured with a standard light microscope. The pixel area of each cell on the image was calculated with ImageJ. Briefly, 8-bit images were thresholded with B\&W default setting and processed with binary functions "Fill Holes" and "Watershed" in sequence. The pixel area of each cell-shaped particle was calculated with "Analyze Particles" function. Only particles within a size range of "80-Infinity" were included to avoid the interference of cell debris. To plot the cell size frequency distribution, pixel areas of at least 100 cells from 4 to 5 random fields (randomly chosen microscopic fields containing at least 20 cells per field) were used to calculate the bin and frequency of the histogram with Data Analysis ToolPak in Excel.

\section{Cell secretome analysis}

$5 \times 10^{5}$ htNSC were seeded in six-well ultra-low attachment plate and cultured for $24 \mathrm{~h} .30 \mu \mathrm{l}$ of Blank or conditioned medium was subjected to SDS-PAGE, and the polyacrylamide gel was stained by silver staining kit (Sigma-Aldrich) according to the manufacturer's instruction. A unique protein band of htNSC condition medium at about $15 \mathrm{kD}$ in size was visually identified, and excised and analyzed by nanoLC-MS/MS using a Waters nanoAcquity and Orbitrap Velos mass spectrometer (Thermo Fisher Scientific) at the proteomic facility of Albert Einstein College of Medicine. Protein identification was performed using Mascot with precursor and product ion tolerances of $25 \mathrm{ppm}$ and $0.4 \mathrm{kD}$, respectively; carbamidometyl Cys as a fixed modification and deamidation (Asn \& GIn), and oxidation (Met) as variable modifications against the SwissProt database.

\section{PTMS antibody generation}

Customized PTMS antibody was collaboratively developed with Pierce Biotechnology, Inc. In brief, several different versions of 26mer short peptides according to the predicted essential regions of PTMS were commercially synthesized and emulsified with Freund's adjuvant to immunize rabbits for generating polyclonal anti-PTMS sera. All anti-PTMS sera were screened together using several methods including Western blots and ELISA as well as immunostaining for negative controls including various ptms knockout mouse samples and positive controls including HA-tagged PTMS expression in cells and tissues. A specific antibody was identified which was generated against the 26-mer peptide sequence of VEAAAELSAKDLKEKKDKVEEKAGRK which corresponded to the amino acids 6-31 of mouse PTMS. This anti-PTMS serum was subjected to IgG purification and further confirmed after purification for the specificity and quality before being used in this study.

\section{PTMS secretion and transfer assays in vitro}

htNSC were transduced with CMV-ptms-HA versus matched control lentivirus for $24 \mathrm{~h}$, and selected with blasticidin at $1 \mu \mathrm{g} / \mathrm{ml}$ for $7-10 \mathrm{~d}$. The post-selected cells (donor) were then cultured in regular medium for $24 \mathrm{~h}$, and the conditioned medium was added to the acceptor cell culture for various amount of time. The acceptor cells were examined by PTMS or HA immunofluorescence or Western blot to assess the intercellular PTMS transfer efficiency.

\section{Immunostaining}

\section{Cell immunostaining}

Cells were seeded on laminin or polylysine-coated coverslips and fixed with 4\% PFA for $15 \mathrm{~min}$ at room temperature before immunostaining. For cultured htNSC spheres, they were fixed with 4\% PFA for $30 \mathrm{~min}$ at room temperature and equilibrated with $20-30 \%$ sucrose, and cryosections were made at a thickness of $10 \mu \mathrm{m}$. 


\section{Tissue immunostaining}

Mice under anesthesia were transcardially perfused with 4\% PFA, and then the brains and peripheral tissues were removed, PFA-fixed, and equilibrated with $20-30 \%$ sucrose, followed by cryosectioning at a thickness of 10 (brain) or $20 \mu \mathrm{m}$ (peripheral tissues). Cell or tissue specimen were blocked with the serum of the appropriate species, treated with primary antibodies, including rabbit anti-PTMS, rabbit anti-HA (3724; Cell Signaling), mouse anti-Sox2 (MAB2018; R\&D Systems), rabbit anti-Nestin (MAB353; Millipore), mouse anti-NeuN (MAB377; Millipore), and mouse anti-HuC/D (A21271; Invitrogen), subsequently incubated with Alexa Fluor 488 or 555-conjugated secondary antibodies. Technical controls for antibody reaction included using naive IgGs of the appropriate species. DAPI staining of sections was used to reveal all cells. Images were captured using Leica SP8 confocal microscope.

\section{Western blot and immunoprecipitation}

Tissue protein lysate was prepared by sonication in ice-cold radioimmunoprecipitation assay (RIPA) lysis buffer (20 mM Tris$\mathrm{HCl}, \mathrm{pH} 7.4,10 \mathrm{mM} \mathrm{NaCl}, 1 \mathrm{mM}$ EDTA, 0.01\% SDS, 1\% Triton X-100, and $1 \times$ protease inhibitor cocktail). CSF was collected from anaesthetized mice by penetrating a pulled capillary tube into the cisterna magna through dura mater, as previous described (Zhang et al, 2017). Serum was prepared by collecting supernatant from fully clotted whole blood ( $1 \mathrm{~h}$ undisturbed at room temperature). CSF and whole blood was mixed with protease inhibitor cocktail immediately upon collection. The boiled protein samples were separated by $18 \%$ SDS-PAGE and were transferred onto $0.2 \mu \mathrm{m}$ polyvinylidene difluoride membrane (PVDF) membrane in acidic buffer (20 mM sodium acetate buffer, pH 5.0) at 15V for overnight. The membranes were then fixed with $0.5 \%$ PFA in PBS for $15 \mathrm{~min}$, washed with $50 \mathrm{mM}$ glycine in PBS for $5 \mathrm{~min}$, and blocked with $5 \%$ non-fat milk in TBST before immunostaining. Primary antibodies were rabbit anti-PTMS (1:2,000), rabbit anti- $\beta$-Actin (1:1,000, \#4967; Cell Signaling), rabbit anti-HA (1:2,000, \#3724; Cell Signaling) and rabbit anti-Transferrin (\#NBP1-97472; Novus Biologicals) primary antibodies overnight at $4^{\circ} \mathrm{C}$, followed by HRP-conjugated goat anti-rabbit secondary antibody (1:10,000; Cell Signaling). For immunoprecipitation, anti-PTMS antibody-coupled Sepharose beads were prepared as described in literature (Michielsen et al, 2020). Briefly, $100 \mathrm{mg}$ protein A-magnetic Sepharose beads (\#28951378; GE Healthcare) were washed with PBS and blocked with $1 \mathrm{~g} / \mathrm{l}$ BSA (Sigma-Aldrich) for $1 \mathrm{~h}$ at room temperature, followed by incubation with $70 \mu \mathrm{g}$ PTMS antibody dissolved in blocking buffer for $1 \mathrm{~h}$ at room temperature with rotation. After three washes with excessive PBS, the beads were cross-linked in $20 \mathrm{mmol} / \mathrm{l}$ dimethyl pimelimidate (Sigma-Aldrich) and $200 \mathrm{mmol} / \mathrm{l}$ triethanolamine (Alfa Aesar) in PBS solution ( $\mathrm{pH} \mathrm{8.6)} \mathrm{for} 30 \mathrm{~min}$ and washed with 200 $\mathrm{mmol} / \mathrm{l}$ triethanolamine (Alfa Aesar) in PBS solution. After two additional repeats of cross-linking and washing, the beads were incubated in $50 \mathrm{mmol} / \mathrm{l}$ ethanolamine (Alfa Aesar) in PBS for $1 \mathrm{~h}$ at room temperature, and two washes with $1 \mathrm{~mol} / \mathrm{l}$ glycine- $\mathrm{HCl}(\mathrm{pH} 3.0)$ at $56^{\circ} \mathrm{C}, 20$ min each. PTMS immunoprecipitation: serum, CSF or cell culture supernatant were mixed with anti-PTMS antibody coupled beads (1:25 for serum and CSF, 1:250 for cell culture supernatant, dry weight), and were rotated in PBST containing $1 \times$ proteinase inhibitor cocktail and $7 \mathrm{~g} / \mathrm{l}$ nonfat milk for overnight at $4^{\circ} \mathrm{C}$. Eluted proteins were SDS-PAGE separated and detected by Western blot as previously described. Ponceau S staining (\#P7170; Sigma-Aldrich) of membrane or Coomassie G-250 staining (\#161-0786; Bio-Rad) (\#AG25; Sigma-Aldrich) of a duplicate gel was used as loading control.

\section{RNA assays}

\section{RNA in situ hybridization}

A $16 Z Z$ probe named Mm-ptms targeting 2-1,059 of NM_026988.2 was designed by Advanced Cell Diagnostics, and in situ detection of ptms mRNA transcription of fixed cyrostat sections using the RNAscope kit (Advanced Cell Diagnostics) was performed according to the manufacturer's protocol. Positive control probe targeting housekeeping gene Pplb and negative control probe targeting bacterial DapB gene were included in each experiment, Images were captured using Leica SP8 confocal microscope.

\section{RNA extraction and $q R T-P C R$}

Total RNA from cells or tissue was extracted using TRIzol (Invitrogen) following the manual. cDNA was synthesized using the Moloney Leukemia Virus Reverse Transcriptase system (Promega). Real-time PCR was performed using the SYBR Green PCR Master Mix (Applied Biosystems). Relative gene expression levels were normalized against the mRNA levels of the house-keeping gene Actb.

\section{Mouse models}

C57BL/6J mice were obtained from Jackson Laboratories and kept in standard, infection-free housing conditions, with 12 light/12 dark cycles and 4-5 mice per cage. The Ptms knockout mouse line was generated by Einstein's transgenic core using CRISPR technology. All procedures were approved by the Institutional Animal Care and Use Committee of the Albert Einstein College of Medicine (protocol \#20171210, \#20171209, and \#20171208).

\section{Lentivirus injection}

The following coordinates were used to target the hypothalamus (AP -1.7, ML \pm 0.25 , DV -5.8), the hypothalamic third ventricle (AP -1.7, $M L 0, D V-5.0$ ), the hippocampus (AP $-1.8, M L \pm 1.3$, DV -1.5), and the parietal cortex (AP -1.8, ML $\pm 2.0, D V-0.2)$. Lentiviruses in the dose of $200 \mathrm{ng}$ p24/ $\mu \mathrm{l}$ with artificial CSF (aCSF; Tocris Bioscience) were injected into brain regions with an ultra-precise stereotactic apparatus (David Kopf Instruments) via a 26-gauge guide cannula and a 33-gauge internal injector (Plastics One) connected to a $5-\mu \mathrm{l}$ Hamilton syringe and infusion pump (WPI Instruments) (Zhang et al, 2017), all brain parenchyma viral injection volumes were $1-\mu \mathrm{l}$ per injection site at rate, and the third ventricle injection and its comparative cortex injection volume was $0.4 \mu \mathrm{l}$ per mouse.

\section{Behavioral tests}

All behavior tests were performed in the behavioral testing room. An Anymaze video-tracking system (Stoelting) equipped with a digital camera connected to a computer was used for the whole course of animal activities in training and experimental sessions of 
behavioral tests as we described previously (Zhang et al, 2013), with minor modifications, including the following tests. (1) The grip test (muscle endurance): as described, a mouse was lifted by the tail and placed on a homemade square grid ( $1 \mathrm{~cm}$ mesh size), the grid was then inverted $30 \mathrm{~cm}$ over a soft pad, and the mouse was allowed to hang by paws for $5 \mathrm{~min}$. Three repeats were performed for each mouse with at least 10 min rest between each trial. (2) Open field test: a mouse was placed into the lower right corner of a white plastic chamber $(40 \mathrm{~cm}$ length $\times 40$ width and $\times 40 \mathrm{~cm}$ height $)$ facing to wall. The mouse was allowed to freely explore the chamber, and locomotor activity was monitored for $10 \mathrm{~min}$. (3) Novel object recognition (Leger et al, 2013): a mouse was allowed to freely explore an open-field box for 10 min before experimental sessions. During the first session (familiarization session), the mouse was allowed to freely explore two similar objects, and during the second session (test session), one of the two objects was replaced by a novel object for $10 \mathrm{~min}$. An intersession interval of 6 - $\mathrm{h}$ between the two sessions were applied. The amount of time that the mouse spent on exploring each object was recorded. A preference index was calculated using the ratio of the amount of time spent exploring any one of the two objects (including novel one) over the total time spent exploring both objects. (4) Y maze spontaneous alternation (Miedel et al, 2017): testing occurred in a Y-shaped maze with three plastic arms at a $120^{\circ}$ angle from each other. A mouse was placed into one arm facing the center junction zone, and allowed to freely explore the maze for $10 \mathrm{~min}$. An arm entry occurred when all four paws of the mouse cross the arm-center zone boundary with the mouse's snout oriented towards the end of the arm. A spontaneous alteration occurred when the mouse made three consecutive arm entries of the $Y$ maze clockwise or counterclockwise. Spontaneous alternation $=$ number of spontaneous alternations/(total number of arm entries - 2). (5) Social affiliation and social recognition (Kaidanovich-Beilin et al, 2011): the testing occurred in a plastic three-chamber neutral box cage (plexiglas, $60 \mathrm{~cm}$ length, $40 \mathrm{~cm}$ width, and $22 \mathrm{~cm}$ height). Each mouse was allowed to explore freely for $5 \mathrm{~min}$ in the cage to habituate to the testing conditions (adaptation). During the social affiliation session, a new mouse (Stranger 1) was placed in a wire containment cup that was located in one side chamber. Subject mouse was allowed free access to explore each of the three chambers for $10 \mathrm{~min}$. During the social recognition session, a second new mouse (Stranger 2) placed to a wire containment cup was put in the opposite side chamber. The subject mouse freely explored each of the three chambers for 10 min during two sections. The time spent in social interaction (sniffing) was recorded. (6) Nest building test: the procedure and rating scale were performed as literature (Deacon, 2006). Briefly, the test used pressed cotton wafers as nestlet, transfer the mice to individual testing cage $1 \mathrm{~h}$ before the dark phase, place one nestlet in each cage but no other environmental enrichment items. Assess the nests the next morning on a rating scale of 1-5 based on literature (Deacon, 2006).

\section{Statistics and reproducibility}

All measured data were presented as mean \pm SEM. Sample sizes of mouse neurobehavioral experiments were designed according to our published studies or literature. All cell biology and molecular biology experiments were repeated independently at least twice. Two-tailed unpaired $t$ test was used for analyses which involved only two groups for comparison, and ANOVA and appropriate post hoc tests were used for analyses which involved more than two groups for comparisons. Software for performing statistics included Prism and Statistical Package for the Social Sciences software (SPSS), and P-value of less than 0.05 was considered statistically significant.

\section{Supplementary Information}

Supplementary Information is available at https://doi.org/10.26508/lsa. 202000917.

\section{Acknowledgements}

The authors sincerely thank Cai laboratory personnel for providing technical supports. This study is supported National Insitutes of Health AG031774, DK099136, HL147477, and DK121435 (all to D Cai). Ethical statement: All procedures of animal studies were approved by the Institutional Care and Use Committee of Albert Einstein College of Medicine (protocol \#20171210, \#20171209, and \#20171208)

\section{Author Contributions}

B Yu: data curation, formal analysis, validation, investigation (performance), methodology (approch development), approaches (cell culture, molecular biology, immunostaining, histology, animal behavioral assays, antibody validation, and animal maintenance), and writing assistance. Y Tang: data curation, formal analysis, validation, investigation (performance), methodology (approch development), and approaches (coculture setup, protein secretome screening, antibody generation, and validation, knockout model generation).

D Cai: conceptualization, resources, research design (hypotheses, specific aims, and strategies), data curation, formal analysis, supervision, funding acquisition, validation, investigation (mentoring, analysis, and evaluation), visualization, methodology (strategies and designs), project administration, writing-original draft, review, editing, and finalizing.

\section{Conflict of Interest Statement}

The authors declare that they have no conflict of interest.

\section{References}

Cai D, Khor S (2019) "Hypothalamic microinflammation" paradigm in aging and metabolic diseases. Cell Metab 30: 19-35. doi:10.1016/j.cmet.2019.05.021

Clinton M, Prangou-Lazaridis M, Panneerselvam C, Horecker BL (1989) Prothymosin $\alpha$ and parathymosin: mRNA and polypeptide levels in rodent tissues. Arch Biochem Biophys 269: 256-263. doi:10.1016/00039861(89)90107-0

Deacon RMJ (2006) Assessing nest building in mice. Nat Protoc 1: 1117-1119. doi:10.1038/nprot.2006.170

Haritos AA, Salvin SB, Blacher R, Stein S, Horecker BL (1985) Parathymosin alpha: A peptide from rat tissues with structural homology to 
prothymosin alpha. Proc Natl Acad Sci U S A 82: 1050. doi:10.1073/ pnas.82.4.1050

Kaidanovich-Beilin O, Lipina T, Vukobradovic I, Roder J, Woodgett JR (2011) Assessment of social interaction behaviors. J Vis Exp 25: 2473. doi: $10.3791 / 2473$

Komiyama T, Pan LX, Haritos AA, Wideman JW, Pan YC, Chang M, Rogers I, Horecker BL (1986) The primary structure of rat parathymosin. Proc Natl Acad Sci U S A 83: 1242. doi:10.1073/pnas.83.5.1242

Kondili K, Tsolas O, Papamarcaki T (1996) Selective interaction between parathymosin and histone H1. Eur J Biochem 242: 67-74. doi:10.1111/ j.1432-1033.1996.0067r.x

Kuilman T, Michaloglou C, Mooi WJ, Peeper DS (2010) The essence of senescence. Gene Dev 24: 2463-2479. doi:10.1101/gad.1971610

Leger M, Quiedeville A, Bouet V, Haelewyn B, Boulouard M, Schumann-Bard P, Freret T (2013) Object recognition test in mice. Nat Protoc 8: 2531-2537. doi:10.1038/nprot.2013.155

Li J, Tang Y, Cai D (2012) IKK $\beta$ / NF-KB disrupts adult hypothalamic neural stem cells to mediate a neurodegenerative mechanism of dietary obesity and pre-diabetes. Nat Cell Biol 14: 999. doi:10.1038/ncb2562

Li J, Tang Y, Purkayastha S, Yan J, Cai D (2014) Control of obesity and glucose intolerance via building neural stem cells in the hypothalamus. Mol Metab 3: 313-324. doi:10.1016/j.molmet.2014.01.012

López-Otín C, Blasco MA, Partridge L, Serrano M, Kroemer G (2013) The hallmarks of aging. Cell 153: 1194-1217. doi:10.1016/j.cell.2013.05.039

Michielsen ECHJ, Diris JHC, Hackeng CM, Wodzig WKWH, Van Dieijen-Visser MP (2020) Highly sensitive immunoprecipitation method for extracting and concentrating low-abundance proteins from human serum. Clinical Chem 51: 222-224. doi:10.1373/clinchem.2004.036251

Miedel CJ, Patton JM, Miedel AN, Miedel ES, Levenson JM (2017) Assessment of spontaneous alternation, novel object recognition and limb clasping in transgenic mouse models of amyloid- $\beta$ and tau neuropathology. J Vis Exp 55523. doi:10.3791/55523

Noble EE, Hahn JD, Konanur VR, Hsu TM, Page SJ, Cortella AM, Liu CM, Song MY, Suarez AN, Szujewski CC, et al (2018) Control of feeding behavior by cerebral ventricular volume transmission of melanin-concentrating hormone. Cell Metab 28: 55-68.e57. doi:10.1016/j.cmet.2018.05.001

Okamoto K, Isohashi F (2000) Purification and primary structure of a macromolecular-translocation inhibitor II of glucocorticoid-receptor binding to nuclei from rat liver. Eur J Biochem 267: 155-162. doi:10.1046/j.1432-1327.2000.00987.x
Rittié L, Fisher GJ (2005) Isolation and culture of skin fibroblasts. In Fibrosis Research: Methods and Protocols, Varga J, Brenner DA, Phan SH (eds) pp 83-98. Totowa, NJ: Humana Press.

Satoh A, Brace CS, Rensing N, Cliften P, Wozniak DF, Herzog ED, Yamada KA, Imai S (2013) Sirt1 extends life span and delays aging in mice through the regulation of Nk2 homeobox 1 in the DMH and LH. Cell Metab 18: 416-430. doi:10.1016/j.cmet.2013.07.013

Tang Y, Horikawa I, Ajiro M, Robles Al, Fujita K, Mondal AM, Stauffer JK, Zheng ZM, Harris CC (2013) Downregulation of splicing factor SRSF3 induces p53 $\beta$, an alternatively spliced isoform of p53 that promotes cellular senescence. Oncogene 32: 2792-2798. doi:10.1038/onc.2012.288

Tang Y, Zuniga-Hertz JP, Han C, Yu B, Cai D (2020) Multifaceted secretion of htNSC-derived hypothalamic islets induces survival and antidiabetic effect via peripheral implantation in mice. Elife 9: e52580. doi:10.7554/ elife. 52580

Trompeter H-I, Blankenburg G, Brügger B, Menne J, Schiermeyer A, Scholz M, Söling H-D (1996) Variable nuclear cytoplasmic distribution of the 11.5-kDa zinc-binding protein (Parathymosin- $\alpha$ ) and identification of a bipartite nuclear localization signal. J Biol Chem 271: 1187-1193. doi:10.1074/jbc.271.2.1187

Trompeter H-I, Söling H-D (1992) Cloning and characterisation of a gene encoding the $11.5 \mathrm{kDa}$ zinc-binding protein (parathymosin- $\alpha$ ). FEBS Lett 298: 245-248. doi:10.1016/0014-5793(92)80068-r

Xiao YZ, Yang M, Xiao Y, Guo Q, Huang Y, Li C), Cai D, Luo XH (2020) Reducing hypothalamic stem cell senescence protects against agingassociated physiological decline. Cell Metab 31: 534-548.e5. doi:10.1016/j.cmet.2020.01.002

Zhang G, Li J, Purkayastha S, Tang Y, Zhang H, Yin Y, Li B, Liu G, Cai D (2013) Hypothalamic programming of systemic ageing involving IKK-beta, NF-kappaB and GnRH. Nature 497: 211-216. doi:10.1038/nature12143

Zhang Y, Kim MS, Jia B, Yan J, Zuniga-Hertz JP, Han C, Cai D (2017) Hypothalamic stem cells control ageing speed partly through exosomal miRNAs. Nature 548: 52. doi:10.1038/nature23282

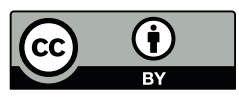

License: This article is available under a Creative Commons License (Attribution 4.0 International, as described at https://creativecommons.org/ licenses/by/4.0/). 